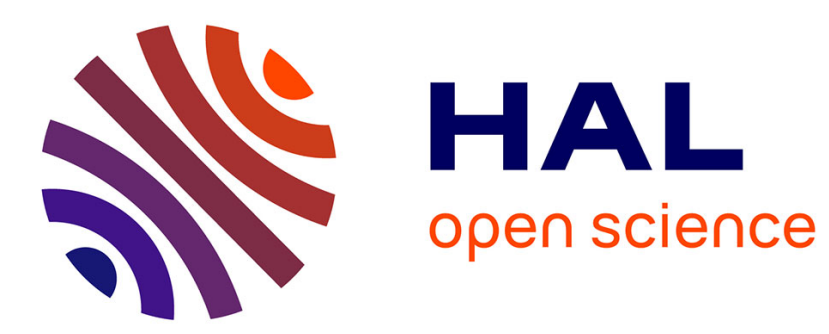

\title{
Batch sizing with controllable production rates in a multi-stage production system
}

Christoph H. Glock

\section{To cite this version:}

Christoph H. Glock. Batch sizing with controllable production rates in a multi-stage production system. International Journal of Production Research, 2011, pp.1. 10.1080/00207543.2010.528058 . hal-00660539

\section{HAL Id: hal-00660539 \\ https://hal.science/hal-00660539}

Submitted on 17 Jan 2012

HAL is a multi-disciplinary open access archive for the deposit and dissemination of scientific research documents, whether they are published or not. The documents may come from teaching and research institutions in France or abroad, or from public or private research centers.
L'archive ouverte pluridisciplinaire HAL, est destinée au dépôt et à la diffusion de documents scientifiques de niveau recherche, publiés ou non, émanant des établissements d'enseignement et de recherche français ou étrangers, des laboratoires publics ou privés. 


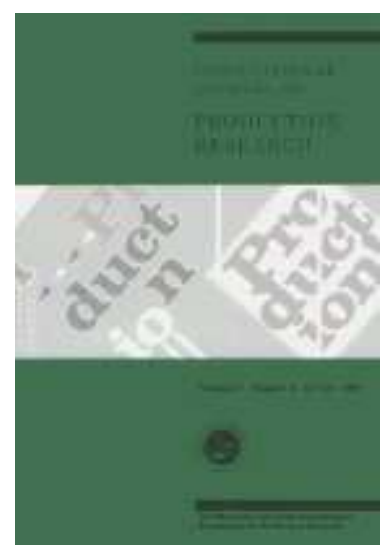

\section{Batch sizing with controllable production rates in a multi- stage production system}

\begin{tabular}{|r|l|}
\hline Journal: & International Journal of Production Research \\
\hline Manuscript ID: & TPRS-2010-IJPR-0165.R2 \\
\hline Manuscript Type: & Original Manuscript \\
\hline $\begin{array}{r}\text { Date Submitted by the } \\
\text { Author: }\end{array}$ & 30-Aug-2010 \\
\hline Complete List of Authors: & $\begin{array}{l}\text { Glock, Christoph; University of Wuerzburg, Chair of Business } \\
\text { Management and Industrial Management }\end{array}$ \\
\hline Keywords: & INVENTORY CONTROL, BATCH SIZING, PRODUCTION PLANNING \\
\hline Keywords (user): & variable production rate, multi-stage lot sizing \\
\hline \multicolumn{2}{|c}{} \\
\hline
\end{tabular}

\section{SCHOLARONE}

Manuscripts 
Batch sizing with controllable production rates in a multi-stage production system

\author{
Christoph H. Glock \\ Chair of Business Management and Industrial Management \\ University of Wuerzburg \\ Sanderring 2 \\ 97070 Wuerzburg \\ Germany \\ Tel.: ++49 9313182408 \\ Fax: ++49931312405 \\ E-Mail: christoph.glock@uni-wuerzburg.de
}

\begin{abstract}
In a recent paper, Glock [2010. Batch sizing with controllable production rates. International Journal of Production Research 20: 5925-5942] studied the impact of a variable production rate on the inventory build-up and the total costs in a two-stage production system. In this paper, we extend Glock's (2010) model to a multi-stage production system where the production rate at each producing stage may be varied within given limits. We compare our model to the classical case with a fixed production rate and show that treating the production rate as a variable is an appropriate measure to reduce excessive inventory in a production system.
\end{abstract}

Keywords: inventory control; production; variable production rate; batch sizing; multi-stage lot-sizing 


\section{Introduction}

The control of inventories in production and supply chain systems has frequently been the subject of interest for researchers in recent years. Reducing excessive inventory in a supply chain may increase the flexibility of the chain and simultaneously reduce the costs associated with producing, storing, and transporting products (see for example Lee et al. 1997). While early works on inventory control mainly focused on production systems with a single production and a single consumption stage, more recent publications have analysed interdependencies between multiple stages of a production system (see e.g. Bogaschewsky et al. 2001; Chang and Chiu 2005; Glock 2010b).

In a recent paper, Glock (2010a) studied the impact of variable production rates on the buildup of inventory in a two-stage production system and showed that deviating from the 'design production rate' of the system may reduce the system's total costs. This is important, as varying the production rate gives production planners flexibility in smoothing material flows and in avoiding the accumulation of inventory at bottleneck stations. The findings of Glock (2010a), however, are not applicable to multi-stage production systems because of the interdependencies between consecutive stages that are neglected in inventory models which consider only two stages. This paper extends the model of Glock (2010a) to a multi-stage production system where the production rates at the stages may be varied between minimum and maximum values.

This paper relates two streams of research, which are: (1) multi-stage production systems and (2) variable production rates in inventory models. Studies that fall along the first stream analysed the impact of different transportation strategies on the system's total costs. For example, Szendrovits (1975) presented a multi-stage production system and assumed that batches of equal sizes are transferred between subsequent stages. He showed that the overlapping of production cycles at subsequent production stages may reduce inventory in the system and lower total costs. Goyal (1976) extended the model of Szendrovits by considering a fixed transportation cost to ship a batch to the next stage, and proposed a solution procedure. Assuming that the number of batches may differ between production stages, Goyal (1977a) and Szendrovits and Drezner (1980) developed solutions for models with equal-sized batch shipments. Drezner et al. (1984) showed that equal-sized batch shipments reduce the system's total costs. Bogaschewsky et al. (2001) finally developed a model where subsequent batch shipments are of unequal size. The complexity of the model required developing algorithms for finding the optimal solution. 


\section{Problem description}

In the following section, we study the case where a single product is manufactured in a serial production system consisting of multiple stages and assume that batch shipments are transferred between subsequent stages. Production systems as the one described in this paper can be found in a variety of application areas, for example in the automotive industry or the electronic industry (see for example Kuo et al. 1996; Cochran and Kim 1998; Chiang et al. 2000). As in Glock (2010a), we differentiate between two types of batch transfer policies that are discussed in Szendrovits (1975) and Goyal (1977b). The first policy suggests transferring batches of equal sizes between subsequent stages, which allows for an overlap between production and consumption stages thus reducing the manufacturing cycle time and the inventory levels in the system. The second policy suggests transferring batches of increasing or decreasing sizes between subsequent stages. The increase or decrease in the batch size is constant and equal to the ratio of the production rate to the demand rate. Both policies have 
been studied in multi-stage production systems (see for example Szendrovits 1975; Bogaschewsky et al. 2001) and will be investigated for variable production rates in the following section.

The following assumptions and notations are used to formulate the problem:

\section{Assumptions:}

1. The model's input parameters are deterministic and constant over time.

2. A uniform lot of size $Q$ is manufactured at each stage.

3. The production rate at each stage can be varied between given limits $p_{s, \min }$ and $p_{s, \max }$.

4. The minimum production rate at each stage exceeds the demand rate and $p_{s, \min }>d$.

5. The unit production costs are a function of the production rate.

6. The unit production cost function is convex in $p_{s}$ and $p_{s}{ }^{0}$ is the 'design production rate' that minimises unit production costs at stage $s$.

7. Batches are only sent to the subsequent stage when the inventory level at this stage reaches zero.

8. The number of batches is identical for each stage.

9. We explicitly focus on a time weighted cost function to enhance the practical applicability of our model. As Higgins et al. (1996) note, production plans are often made with planning horizons rolling through time as time progresses. Thus, companies who wish to implement our model as a heuristic planning tool may specify a planning period and repeat the planning process after the planning time has elapsed. Note that this assumption is not uncommon in the literature, cf. for example Bogaschewsky et al. (2001) and Glock (2010a). However, normalizing the planning period to 1 time unit results in the common money-per-unit-time cost function.

10. Shortages are not allowed.

\section{Notations:}

$c_{s}\left(p_{s}\right)=$ unit production cost function of stage $s$

$A_{s}=$ setup costs per setup at stage $s$

$T_{s}=$ transportation costs per shipment at stage $s$

$d=$ demand rate in units per unit time

$D=$ total demand in the planning period

$h_{s}=$ inventory carrying charges per unit per unit of time at stage $s$ 
$q_{j, s}=$ size of the $j$ th batch produced at stage $s$

$\lambda_{s}=$ the proportional increase/decrease in successive shipments within a batch production run at stage $s$

$m=$ number of batch shipments in a production cycle

$p_{s}=$ production rate in units per unit of time at stage $s$ with $p_{S+1}=d$

$p_{s}{ }^{0}=$ design production rate at stage $s$

$a_{s, i}=$ cost parameters of the unit production cost function

$Q=$ production lot size

$S=$ number of stages

\section{Definitions:}

$I_{s}^{\text {reg }}=$ 'regular' inventory at stage $s$

$I_{S}^{\text {wait }}=$ inventory due to waiting times at stage $s$

$I C=$ inventory carrying costs

$P C=$ production costs

$T C=$ total costs in the planning period

$T W I=$ time weighted inventory

$|a|=$ the absolute value of $a$, i.e. $|a|=\sqrt{a^{2}}$

\section{Model formulation and solution}

\section{The rigid case}

\section{a) Equal-sized batch shipments}

The first scenario we analyse is the case where equal-sized batch shipments are transported to the subsequent stage and where the production rate may be varied only before the start of a production run. The corresponding inventory time plots are illustrated exemplarily in figure 1 (cf. the bold lines. Note that the dashed lines represent the case of a reduced production rate). It is shown that a lot of size $Q$ is manufactured at stage $s$ and that $m$ batches of size $Q / m$ are transported to the subsequent stage whenever the inventory level in front of the stage drops to zero. Stage $s+1$ consumes the products delivered by stage $s$ and produces a product which is then stored and successively shipped batch-wise to stage $s+2$, which is the final stage in the example. Note that our model is valid for an arbitrary number of stages and is not restricted to three stages as in figure 1 . In the example, it can be seen that due to $p_{s+1}>p_{s+2}$, the second 
batch is finished at stage $s+1$ before the first batch is completely used up at stage $s+2$, wherefore it has to be kept in stock for $t_{w, s+1,1}=t_{p, s+2}-t_{p, s+1}$ time units. In contrast, due to $p_{s+1}$ $>p_{s}$, the production time of a batch at stage $s$ exceeds the consumption time of a batch at stage $s+1$, wherefore batches $1, \ldots, m-1$ have to be kept in stock for $t_{w, s, i}$ time units before they are sent to the subsequent stage in order to build up enough inventory to assure an uninterrupted supply of materials. Further, the example illustrates that a change in the production rate at stage $s+1$ may influence inventory build-up at stage $s+1$ as well as at the preceding and the succeeding stage (cf. the dashed lines in figure 1).

Figure 1

As described in Glock (2010a), the time weighted inventory at stage $s+1$ consists of a 'regular' inventory, which corresponds to the triangles shown in the second plot in figure 1, and inventory due to waiting times. The 'regular' inventory can be expressed as follows:

(1) $I_{s}^{r e g}=\frac{Q^{2}}{2 m}\left(\frac{1}{p_{s}}+\frac{1}{p_{s+1}}\right)$

Inventory due to waiting times can be calculated as:

(2a) $\quad I_{s}^{\text {wait }}=\frac{Q^{2}}{m^{2}} \sum_{i=1}^{m-1} i\left(\frac{1}{p_{s+1}}-\frac{1}{p_{s}}\right)=\frac{Q^{2}}{2 m}(m-1)\left(\frac{1}{p_{s+1}}-\frac{1}{p_{s}}\right)$ for $p_{s}>p_{s+1} \quad$ and

(2b) $I_{s}^{\text {wait }}=\frac{Q^{2}}{m^{2}} \sum_{i=1}^{m-1} i\left(\frac{1}{p_{s}}-\frac{1}{p_{s+1}}\right)=\frac{Q^{2}}{2 m}(m-1)\left(\frac{1}{p_{s}}-\frac{1}{p_{s+1}}\right)$ for $p_{s+1}>p_{s}$

From (2a) and (2b) a general expression can be formulated as follows:

$$
I_{s}^{\text {wait }}=\frac{Q^{2}}{2 m}(m-1)\left|\frac{1}{p_{s+1}}-\frac{1}{p_{s}}\right|
$$

The time weighted inventory is given as the sum of (1) and (3). The inventory carrying costs in the planning period for all $S$ stages thus equal:

$$
I C=\frac{D Q}{2 m} \sum_{s=1}^{S}\left(\left(\frac{1}{p_{s}}+\frac{1}{p_{s+1}}\right)+(m-1)\left|\frac{1}{p_{s+1}}-\frac{1}{p_{s}}\right|\right) h_{s}
$$

Apart from the inventory carrying costs, the system encounters production costs which vary with a varying production rate. In the following, we assume that the unit production cost function is of the form specified by Eiamkanchanalai and Banerjee (1999) and used in Glock (2010a), wherefore we can conclude that:

$$
P C_{s}=D c_{s}\left(p_{s}\right)=D\left(a_{s, 0} p_{s}^{2}-a_{s, 1} p_{s}+a_{s, 2}\right)
$$


The total costs consist of the sum of (4) and (5) and the setup and transportation costs in the planning period, which amount to $\left(A_{s}+m T_{s}\right) D / Q$. Thus, it follows that:

$$
T C=\frac{D Q}{2 m} \sum_{s=1}^{S}\left(\left(\frac{1}{p_{s}}+\frac{1}{p_{s+1}}\right)+(m-1) \frac{1}{p_{s+1}}-\frac{1}{p_{s}} \mid\right) h_{s}+\sum_{s=1}^{S}\left(A_{s}+m T_{s}\right) \frac{D}{Q}+\sum_{s=1}^{S} D c_{s}\left(p_{s}\right)
$$

It can be easily shown that (6) is convex in $Q$ for a given $p$-vector and given values for $m$. Thus, the optimal solution for $Q$ is given as:

$$
Q^{*}=\sqrt{\frac{2 m \sum_{s=1}^{S}\left(A_{s}+m T_{s}\right)}{\sum_{s=1}^{S}\left(\left(\frac{1}{p_{s}}+\frac{1}{p_{s+1}}\right)+(m-1)\left|\frac{1}{p_{s+1}}-\frac{1}{p_{s}}\right|\right) h_{s}}}
$$

Substituting (7) in (6), the total cost function reduces to:

$$
T C=D \sqrt{\frac{2}{m} \sum_{s=1}^{S}\left(\left(\frac{1}{p_{s}}+\frac{1}{p_{s+1}}\right)+(m-1)\left|\frac{1}{p_{s+1}}-\frac{1}{p_{s}}\right|\right) h_{s} \sum_{s=1}^{S}\left(A_{s}+m T_{s}\right)}+\sum_{s=1}^{S} D c_{s}\left(p_{s}\right)
$$

As could be shown by Szendrovits and Drezner (1980), (8) is quasi-convex in $m$. Thus, for a given $p$-vector, an optimal solution for $m$ may be calculated with the help of the following optimality condition:

\section{(9) $\quad T C\left(m^{*}-1\right) \geq T C\left(m^{*}\right) \leq T C\left(m^{*}+1\right)$}

The cost function shown in (8) was analysed by Glock (2010a) for the case $S=2$ and $p_{s}>$ $p_{s+1}$, who derived rules of how to vary the production rate in order to reduce total costs. However, his results may only partially be transferred to the case of a multi-stage production system due to the interdependencies that arise between multiple production stages. The basic problem associated with varying production rates in a serial production system is illustrated in figure 2, which considers three production stages and two buffer stocks which are located between each two successive stages. If we consider the case where $p_{s-1}>p_{s}<p_{s+1}$, inventory accumulates in the buffer stock in front of stage $s$ since stage $s$ is not able to consume the products delivered by the preceding stage as fast as stage $s-1$ produces them, and in the buffer stock in front of stage $s+1$ since a certain amount of inventory has to be build up prior to the start of the production cycle at stage $s+1$ in order to assure an uninterrupted supply of materials. If we now assume that the production rate is increased at stage $s$ such that $p_{s-1}>p_{s}$ $>p_{s+1}$, inventory is reduced in the first buffer stock and partially transferred to the second (depending on the former and actual ratio of $p_{s} / p_{s+1}$ ). Thus, it becomes obvious that varying the production rate at any particular stage may influence upstream and downstream inventory, which is helpful in smoothing material flows and in exploiting different inventory carrying charges at different stages in the production system. 
Figure 2

The basic problem associated with optimizing (8) in $p_{s}$ is that (8) is not necessarily convex in $p_{s}$ for given values of $m$. However, we may state the following theorems to derive a solution:

Theorem 1: For $m=1$, producing with $p_{s}<p_{s}{ }^{0}$ cannot be optimal.

Proof: see appendix 1.

Theorem 2: For $m=2$, it is optimal to produce with $p_{s}=p_{s}{ }^{0}$ in case $p_{s-1}<p_{s}>p_{s+1}$ and to increase $p_{s}$ in all other instances.

Proof: see appendix 1.

Theorem 3: For $m>2$,

- producing with $p_{s}<p_{s}^{0}$ cannot be optimal in case $p_{s-1}>p_{s}>p_{s+1}$ and $m /(m-2)>h_{s} / h_{s-1}$. If $p_{s-1}>p_{s}>p_{s+1}$ and $m /(m-2)<h_{s} / h_{s-1}$, producing with $p_{s}>p_{s}{ }^{0}$ cannot be optimal.

- producing with $p_{s}>p_{s}{ }^{0}$ cannot be optimal in case $p_{s-1}<p_{s}>p_{s+1}$.

- producing with $p_{s}<p_{s}{ }^{0}$ cannot be optimal in case $p_{s-1}>p_{s}<p_{s+1}$.

- producing with $p_{s}<p_{s}^{0}$ cannot be optimal in case $p_{s-1}<p_{s}<p_{s+1}$ and $m /(m-2)>h_{s-1} / h_{s}$. If $p_{s-1}>p_{s}>p_{s+1}$ and $m /(m-2)<h_{s-1} / h_{s}$, producing with $p_{s}>p_{s}{ }^{0}$ cannot be optimal.

Proof: see appendix 1.

To calculate a good, but not necessarily optimal value for $p_{s}$, we can apply a one-dimensional search algorithm. For example, we could conduct a grid search and, starting from $p_{\min }$, increase $p$ stepwise until $p_{\max }$ is reached and select the best solution found during the search process as the final solution (see Gill et al. (1986) for other alternatives). The following procedure can be used to find a solution for $m^{*}, p_{s}^{*}$, and $Q^{*}$ :

Step 1: Set $m=1$ and search $p_{s} \in\left[p_{\mathrm{s}}{ }^{0} ; p_{s, \max }\right] \forall s$ that minimises $T C$. Set $T C^{*}=T C, m^{*}=m$, and $p_{s}^{*}=p_{s} \forall s$.

Step 2: Set $m=2$ and $p_{s}=p_{s}{ }^{0} \forall s$ where $p_{s-1}<p_{s}>p_{s-1}$. Otherwise, search $p_{s} \in\left[p_{\mathrm{s}}{ }^{0} ; p_{s, \max }\right]$ that minimises $T C$. If $T C>T C^{*}$, Goto Step 5 .

Set $T C^{*}=T C, m^{*}=m, p_{s}{ }^{*}=p_{s} \forall s$, and $m=m+1$ 
Step 3: Search $p_{s} \in\left[p_{s, \min } ; p_{s}^{0}\right]$ or $p_{s} \in\left[p_{s}{ }^{0} ; p_{s, \max }\right]$ that minimises $T C$ according to the conditions specified above. If $T C>T C^{*}$, Goto Step 5.

Step 4: Set $T C^{*}=T C, m^{*}=m, p_{s}^{*}=p_{s} \forall s$, and $m=m+1$. Goto Step 3 .

Step 5: Find $Q^{*}$ from (6).

\section{b) Unequal-sized batch shipments}

In the case where unequal-sized batches are shipped to the subsequent stage, the size of the $j$ th batch at stage $s$ can be calculated as follows (see Goyal 1977b):

$$
q_{j, s}=q_{1, s} \lambda_{s}^{j-1}=q_{1, s}\left(p_{s} / p_{s+1}\right)^{j-1} \quad \text { with } Q=\sum_{i=1}^{m} q_{i, s}=q_{1, s} \sum_{i=1}^{m} \lambda_{s}^{i-1}
$$

It is obvious that subsequent batches increase in size in case $p_{s}>p_{s+1}$ and decrease if $p_{s}<p_{s+1}$. The corresponding inventory time plots that result if $t_{p, i, s}=t_{p, i-1, s+1}$ are illustrated in figure 3 (cf. the bold lines. The dashed lines again illustrate the case of a reduced production rate). As can be seen, there is no inventory due to waiting times if subsequent batches increase or decrease by $\lambda_{s}=p_{s} / p_{s+1}$.

\section{Figure 3}

The time weighted inventory at stage $s$ can be calculated as follows:

$$
\begin{aligned}
T W I_{s} & =\frac{q_{1, s}}{2}\left(\frac{q_{1, s}}{p_{s}}+\frac{q_{1, s}}{p_{s+1}}\right)+\frac{q_{2, s}}{2}\left(\frac{q_{2, s}}{p_{s}}+\frac{q_{2, s}}{p_{s+1}}\right)+\ldots+\frac{q_{m, s}}{2}\left(\frac{q_{m, s}}{p_{s}}+\frac{q_{m, s}}{p_{s+1}}\right)= \\
& =\frac{q_{1, s}^{2}}{2}\left(\frac{1}{p_{s}}+\frac{1}{p_{s+1}}\right) \sum_{i=1}^{m}\left(\lambda_{s}^{i-1}\right)^{2}=\frac{Q^{2}}{2}\left(\frac{1}{p_{s}}+\frac{1}{p_{s+1}}\right) \frac{\left(\lambda_{s}^{m}+1\right)\left(\lambda_{s}-1\right)}{\left(\lambda_{s}+1\right)\left(\lambda_{s}^{m}-1\right)}
\end{aligned}
$$

The inventory carrying costs can now be derived if the time weighted inventory is summed up over all $S$ stages and if the resulting expression is multiplied with the number of lots in the planning period $(D / Q)$ and the unit inventory carrying charges per unit of time $h_{s}$. Further considering setup costs, transportation costs, and production costs leads to the total cost function:

$$
T C=\frac{D Q}{2} \sum_{s=1}^{S}\left(\frac{1}{p_{s}}+\frac{1}{p_{s+1}}\right) \frac{\left(\lambda_{s}^{m}+1\right)\left(\lambda_{s}-1\right)}{\left(\lambda_{s}+1\right)\left(\lambda_{s}^{m}-1\right)} h_{s}+\sum_{s=1}^{S}\left(A_{s}+m T_{s}\right) \frac{D}{Q}+\sum_{s=1}^{S} D c_{s}\left(p_{s}\right)
$$

Since (12) is convex in $Q$ for given values for $m$ and $p_{s}$, a solution for the optimal order quantity (and with (10) for the optimal size of the first batch) can be calculated with the help of differential calculus. It follows: 


$$
Q^{*}=\sqrt{\frac{2 \sum_{s=1}^{S}\left(A_{s}+m T_{s}\right)}{\sum_{s=1}^{S}\left(\frac{1}{p_{s}}+\frac{1}{p_{s+1}}\right) \frac{\left(\lambda_{s}^{m}+1\right)\left(\lambda_{s}-1\right)}{\left(\lambda_{s}+1\right)\left(\lambda_{s}^{m}-1\right)} h_{s}}}
$$

Substituting (13) in (12), the total cost function reduces to:

$$
T C=D \sqrt{2 \sum_{s=1}^{S}\left(\frac{1}{p_{s}}+\frac{1}{p_{s+1}}\right) \frac{\left(\lambda_{s}^{m}+1\right)\left(\lambda_{s}-1\right)}{\left(\lambda_{s}+1\right)\left(\lambda_{s}^{m}-1\right)} h_{s} \sum_{s=1}^{S}\left(A_{s}+m T_{s}\right)}+\sum_{s=1}^{S} D c_{s}\left(p_{s}\right)
$$

Since it may be shown that (14) is convex in $m$ for given values of $p_{s}$, an optimal solution may be derived with the help of the following optimality condition:

$$
T C\left(m^{*}-1\right) \geq T C\left(m^{*}\right) \leq T C\left(m^{*}+1\right)
$$

However, (14) is not necessarily convex in $p_{s}$. To derive a solution for the production rate, we state the following theorem:

Theorem 4: For $m=1$, producing with $p_{s}<p_{s}{ }^{0}$ cannot be optimal.

Proof: follows directly from theorem 1.

Further, simulation studies indicated that (14) is quasi-convex in $p_{s}$. To calculate a good, but not necessarily optimal value for $p_{s}$, we may thus again apply a one-dimensional search algorithm over the intervals $\left[p_{s, \text { min }} ; p_{s, \text { max }}\right]$ or $\left[p_{s}^{0} ; p_{s, \text { max }}\right]$, respectively. The following procedure can be used to find a solution for $m^{*}, p_{s}^{*}$, and $Q^{*}$ (or $q_{1, s}{ }^{*}$, respectively):

Step 1: Set $m=1$ and search $p_{s} \in\left[p_{s}^{0} ; p_{s, \max }\right]$ that minimises $T C$. Set $T C^{*}=T C, m^{*}=m$, and $p_{s}^{*}=p_{s}$. Set $m=m+1$.

Step 2: Search $p_{s} \in\left[p_{s, \min } ; p_{s, \max }\right]$ that minimises $T C$. If $T C>T C^{*}$, Goto Step 4 .

Step 3: Set $T C^{*}=T C, m^{*}=m, p_{s}^{*}=p_{s}$, and $m=m+1$. Goto Step 2 .

Step 4: Find $Q^{*}$ from (13) and $q_{1, s} *$ from (10).

\section{The flexible case}

a) Equal-sized batch shipments

In the previous section, it was assumed that the production rate has to be fixed before a production run starts. In the following, we relax this assumption and suppose that each batch may be produced with a separate production rate. In compliance with earlier work, we assume that the costs of changing the production rate are negligible. 
If each batch is manufactured with a separate production rate, the 'regular' inventory given in (1) has to be reformulated as follows:

$$
I_{s}^{r e g}=\frac{Q^{2}}{2 m^{2}} \sum_{i=1}^{m}\left(\frac{1}{p_{i, s}}+\frac{1}{p_{i, s+1}}\right)
$$

Similarly, inventory due to waiting times can be calculated as:

$$
\begin{aligned}
& I_{s}^{\text {wait }}=\frac{Q}{m} \sum_{i=2}^{m}\left(\sum_{j=1}^{i-1} \frac{Q}{m p_{j, s+1}}-\sum_{j=2}^{i} \frac{Q}{m p_{j, s}}\right)=\frac{Q^{2}}{m^{2}} \sum_{i=2}^{m}\left(\sum_{j=1}^{i-1} \frac{1}{p_{j, s+1}}-\sum_{j=2}^{i} \frac{1}{p_{j, s}}\right) \quad \text { for } p_{s}>p_{\mathrm{s}+1} \quad \text { and } \\
& I_{s}^{\text {wait }}=\frac{Q}{m} \sum_{i=2}^{m}\left(\sum_{j=i}^{m} \frac{Q}{m p_{j, s}}-\sum_{j=i-1}^{m-1} \frac{Q}{m p_{j, s+1}}\right)=\frac{Q^{2}}{m^{2}} \sum_{i=2}^{m}\left(\sum_{j=i}^{m} \frac{1}{p_{j, s}}-\sum_{j=i-1}^{m-1} \frac{1}{p_{j, s+1}}\right) \quad \text { for } p_{s}<p_{\mathrm{s}+1} \quad \text { and }
\end{aligned}
$$

A general expression can be formulated from (17a) and (17b) as follows:

$$
I_{s}^{\text {wait }}=\frac{Q^{2}}{m^{2}}\left(\operatorname{Max}\left[\sum_{\mathrm{i}=2}^{m} \sum_{j=1}^{i-1} \frac{1}{p_{j, s+1}}, \sum_{\mathrm{i}=2}^{m} \sum_{j=i}^{m} \frac{1}{p_{j, s}}\right]-\operatorname{Min}\left[\sum_{\mathrm{i}=2}^{m} \sum_{j=2}^{i} \frac{1}{p_{j, s}}, \sum_{\mathrm{i}=2}^{m} \sum_{j=i-1}^{m-1} \frac{1}{p_{j, s+1}}\right]\right)
$$

Note that (18) and (3) are identical for $p_{j, s}=p_{k, s} \forall j, k$. The time weighted inventory is again given as the sum of (16) and (18). The inventory carrying costs in the planning period thus equal:

$$
I C=\frac{D Q}{m^{2}} \sum_{s=1}^{S} \delta_{s} h_{s}
$$

where $\delta_{s}=\frac{1}{2} \sum_{i=1}^{m}\left(\frac{1}{p_{i, s}}+\frac{1}{p_{i, s+1}}\right)+\operatorname{Max}\left[\sum_{\mathrm{i}=2}^{m} \sum_{j=1}^{i-1} \frac{1}{p_{j, s+1}}, \sum_{\mathrm{i}=2}^{m} \sum_{j=i}^{m} \frac{1}{p_{j, s}}\right]-\operatorname{Min}\left[\sum_{\mathrm{i}=2}^{m} \sum_{j=2}^{i} \frac{1}{p_{j, s}}, \sum_{\mathrm{i}=2}^{m} \sum_{j=i-1}^{m-1} \frac{1}{p_{j, s+1}}\right]$

Considering production costs and setup and transportation costs leads to the total cost function:

$$
T C=\frac{D Q}{m^{2}} \sum_{s=1}^{S} \delta_{s} h_{s}+\sum_{s=1}^{S}\left(A_{s}+m T_{s}\right) \frac{D}{Q}+\frac{D}{m} \sum_{s=1}^{S} \sum_{i=1}^{m} c_{i, s}\left(p_{i, s}\right)
$$

Since (20) is convex in $Q$ for given values for $m$ and a given $p_{s}$-vector, the optimal solution for $Q$ is given as follows:

$$
Q^{*}=\sqrt{\frac{m^{2} \sum_{s=1}^{S}\left(A_{s}+m T_{s}\right)}{\sum_{s=1}^{S} \delta_{s} h_{s}}}
$$

Substituting (21) in (20) leads to:

$$
T C=\frac{D}{m}\left(2 \sqrt{\sum_{s=1}^{S}\left(A_{s}+m T_{s}\right) \sum_{s=1}^{S} \delta_{s} h_{s}}+\sum_{s=1}^{S} \sum_{i=1}^{m} c_{i, s}\left(p_{i, s}\right)\right)
$$


Since (22) is not necessarily convex in $p_{i, s}$ and $m$, we state the following theorems to derive a good solution:

Theorem 5: In case $p_{s-1}>p_{s}>p_{s+1}$

- it is optimal to increase $p_{1, s}$.

- it is optimal to increase $p_{m, s}$ if $h_{s-1}>h_{s}$ and to reduce $p_{m, s}$ if $h_{s}<h_{s-1}$.

- it is optimal to increase $p_{2, s, \ldots, p_{m-1, s}}$ if $h_{s-1}>h_{s}$ and to reduce $p_{2, s, \ldots, p_{m-1, s}}$ if $h_{s-1}<h_{s}$. Further, it is optimal to vary $p_{j, s}$ more than $p_{k, s}$ for $j<k$.

Proof: see appendix 2.

Theorem 6: In case $p_{s-1}>p_{s}<p_{s+1}$

- $\quad$ it is optimal to increase $p_{\mathrm{j}, s} \forall j$.

- $\quad$ it is optimal to vary $p_{j, s}$ stronger than $p_{k, s}$ for $j<k$ if $h_{s-1}>h_{s}$ and vice versa.

Proof: see appendix 3.

Theorem 7: In case $p_{s-1}<p_{s}>p_{s+1}$

- it is optimal to increase $p_{1, s}$ if $h_{s}>h_{s-1}$ and to reduce $p_{1, s}$ if $h_{s}<h_{s-1}$.

- it is optimal to increase $p_{m, s}$ if $\mathrm{h}_{\mathrm{s}-1}>h_{s}$ and to reduce $p_{m, s}$ if $h_{s-1}<h_{s}$.

- it is optimal to reduce $p_{2, s}, \ldots, p_{m-1, s}$. Further, it is optimal to reduce $p_{j, s}$ stronger than $p_{k, s}$ for $j<k$ if $h_{s-1}>h_{s}$ and vice versa.

Proof: see appendix 4.

Theorem 8: In case $p_{s-1}<p_{s}<p_{s+1}$

- it is optimal to increase $p_{1, s}$ if $h_{s}>h_{s-1}$ and to reduce $p_{1, s}$ if $h_{s}<h_{s-1}$.

- it is optimal to increase $p_{m, s}$.

- it is optimal to increase $p_{2, s}, \ldots, p_{m-1, s}$ in case $h_{s}>h_{s-1}$ and vice versa. Further, it is optimal to vary $p_{j, s}$ stronger than $p_{k, s}$ for $j>k$.

Proof: see appendix 5.

To calculate a good, but not necessarily optimal value for $p_{i, s}$, we can again apply a line search algorithm over the intervals identified above. Further, we calculate a solution for $m$ with the help of the following condition:

(23) $T C\left(m^{*}-1\right) \geq T C\left(m^{*}\right) \leq T C\left(m^{*}+1\right)$

The following procedure can be applied to find a solution for $m^{*}, Q^{*}$, and the $p_{s}$-vector: 
Step 1: Set $m=1$ and search $p_{1, s} \in\left[p_{s}{ }^{0} ; p_{s, \max }\right]$ or $p_{1, s} \in\left[p_{s, \min } ; p_{s}{ }^{0}\right]$, respectively, that minimises $T C$. Set $T C^{*}=T C, m^{*}=m$, and $p_{1, \mathrm{~s}}^{*}=p_{1, \mathrm{~s}} \forall s$. Set $m=m+1$.

Step 2: Search $p_{j, s} \in\left[p_{s, \min } ; p_{s}{ }^{0}\right]$ or $p_{j, s} \in\left[p_{s}{ }^{0} ; p_{s, \max }\right]$ that minimises $T C$ according to the conditions specified above. If $T C>T C^{*}$, Goto Step 4.

Step 3: Set $T C^{*}=T C, m^{*}=m, p_{j, s}^{*}=p_{j, s} \forall j, s$ and $m=m+1$. Goto Step 2.

Step 4: Find $Q^{*}$ from (21).

\section{b) Unequal-sized batch shipments}

In the case where unequal-sized batches are transported to the subsequent stage, the size of the $j$ th batch depends on the size of the first shipment and the production rate of batches 2 to $j$ (cf. figure 3). Thus, it follows that

$$
q_{j, s}=q_{1, s} \prod_{i=2}^{j} \frac{p_{i, s}}{p_{i, s+1}} \quad \text { and } \quad Q=\sum_{j=1}^{m} q_{j, s}=q_{1, s} \sum_{j=1}^{m} \prod_{i=2}^{j} \frac{p_{i, s}}{p_{i, s+1}}
$$

The time weighted inventory at stage $s$ can be calculated as follows (cf. Glock 2010a):

$$
\begin{aligned}
T W I_{s} & =\frac{q_{1, s}}{2}\left(\frac{q_{1, s}}{p_{1, s}}+\frac{q_{1, s}}{p_{1, s+1}}\right)+\frac{q_{2, s}}{2}\left(\frac{q_{2, s}}{p_{2, s}}+\frac{q_{2, s}}{p_{2, s+1}}\right)+\ldots+\frac{q_{m, s}}{2}\left(\frac{q_{m, s}}{p_{m, s}}+\frac{q_{m, s}}{p_{m, s+1}}\right)= \\
& =\frac{q_{1, s}^{2}}{2} \sum_{j=1}^{m}\left(\prod_{i=2}^{j} \frac{p_{i, s}}{p_{i, s+1}}\right)^{2}\left(\frac{1}{p_{j, s}}+\frac{1}{p_{j, s+1}}\right)
\end{aligned}
$$

The inventory costs may again be calculated by summing up the time weighted inventory over all $S$ stages and by multiplying the resulting expression with the number of lots in the planning period $(D / Q)$ and the unit inventory carrying charges per unit of time $h_{s}$. Further considering setup, transportation and production costs leads to the total cost function:

$$
T C=\frac{D Q}{2} \sum_{s=1}^{S} \frac{\sum_{j=1}^{m}\left(\prod_{i=2}^{j} \frac{p_{i, s}}{p_{i, s+1}}\right)^{2}\left(\frac{1}{p_{j, s}}+\frac{1}{p_{j, s+1}}\right) h_{s}}{\left(\sum_{j=1}^{m} \prod_{i=2}^{j} \frac{p_{i, s}}{p_{i, s+1}}\right)^{2}}+\sum_{s=1}^{S}\left(A_{s}+m T_{s}\right) \frac{D}{Q}+D \sum_{s=1}^{S} \sum_{j=1}^{m} \frac{c_{j, s}\left(p_{j, s}\right) \prod_{i=2}^{j} \frac{p_{i, s}}{p_{i, s+1}}}{\sum_{j=1}^{m} \prod_{i=2}^{j} \frac{p_{i, s}}{p_{i, s+1}}}
$$

Since (26) is convex in $Q$ for given values for $m$ and $p_{i, s}$, the optimal solution for $Q$ is given as: 


$$
Q^{*}=\sqrt{\frac{2 \sum_{s=1}^{S}\left(A_{s}+m T_{s}\right)}{\sum_{s=1}^{S} \frac{\sum_{j=1}^{m}\left(\prod_{i=2}^{j} \frac{p_{i, s}}{p_{i, s+1}}\right)^{2}\left(\frac{1}{p_{j, s}}+\frac{1}{p_{j, s+1}}\right) h_{s}}{\left(\sum_{j=1}^{m} \prod_{i=2}^{j} \frac{p_{i, s}}{p_{i, s+1}}\right)^{2}}}}
$$

Substituting (27) in (26) leads to:

$$
T C=D\left(\sqrt{2 \sum_{s=1}^{S} \frac{\sum_{j=1}^{m}\left(\prod_{i=2}^{j} \frac{p_{i, s}}{p_{i, s+1}}\right)^{2}\left(\frac{1}{p_{j, s}}+\frac{1}{p_{j, s+1}}\right)}{\left(\sum_{j=1}^{m} \prod_{i=2}^{j} \frac{p_{i, s}}{p_{i, s+1}}\right)^{2}} \sum_{s=1}^{S}\left(A_{s}+m T_{s}\right)+\sum_{s=1}^{S} \sum_{j=1}^{m} \frac{c_{j, s}\left(p_{j, s}\right) \prod_{i=2}^{j} \frac{p_{i, s}}{p_{i, s+1}}}{\sum_{j=1}^{m} \prod_{i=2}^{j} \frac{p_{i, s}}{p_{i, s+1}}}}\right)
$$

Since (28) is not necessarily convex in $p_{i, s}$ and $m$, we state the following theorem to derive a good solution:

Theorem 9: Producing the first batch with $p_{1, s}<p_{s}^{0}$ cannot be optimal.

Proof: this has been shown in Glock (2010a).

For batches 2 to $m$, a variation in $p_{i, s}$ may both lead to an increase or to a decrease in inventory. This is due to the fact that the variation of the production rate of a particular batch may both influence the size of subsequent batches as well as the transfer of inventory between subsequent buffer stocks. Thus, complex interdependencies arise between the production rates which are used to manufacture the batches at subsequent stages and the inventory carrying cost charges in the buffer stocks between the stages. Therefore, it is difficult to derive further propositions which are valid in general.

To calculate a good, but not necessarily optimal value for $p_{i, s}$, we can again apply a line search algorithm over the intervals $\left[p_{\mathrm{s}}^{0} ; p_{s, \max }\right]$ or $\left[p_{s, \min } ; p_{s, \max }\right]$, respectively. Further, we calculate a solution for $m$ with the help of the following condition:

$$
T C\left(m^{*}-1\right) \geq T C\left(m^{*}\right) \leq T C\left(m^{*}+1\right)
$$

The following procedure can be applied to find a solution for $m^{*}, Q^{*}$, and the $p_{s^{-}}$-vector:

Step 1: Set $m=1$ and search $p_{1, \mathrm{~s}} \in\left[p_{\mathrm{s}}^{0} ; p_{s, \max }\right]$ that minimises $T C$. Set $T C^{*}=T C, m^{*}=m$, and $p_{1, s} *=p_{1, s}$. Set $m=m+1$. 


\section{Table 1}

As shown in table 2, shipping batches of unequal sizes to the subsequent stage reduces total costs, while shipping batches of equal sizes leads to an increase in total costs. Further, it can be seen that shipping unequal-sized batches reduces inventory in all three buffer stocks (note that $I_{s}$ denotes the buffer stock between stages $s$ and $s+1$ ), which is due to the fact that smaller initial shipments enable stages $2, \ldots, S$ to initiate production earlier, which reduces inventory in the system.

The results shown in tables 3 to 6 illustrate that allowing the production rate to be varied reduces total costs. Further, it becomes obvious that the flexible case leads to lower total costs than the rigid case, and that transporting unequal-sized batches always leads to lower costs than the case where batches of equal size are shipped to the subsequent stage. Although the last result is well known from the literature (see for example Goyal 1977b), it has to be considered that implementing unequal-sized batch shipments may lead to difficulties, for example due to problems in standardising transportation equipment. In such a case, transferring batches of unequal size from one stage to the next may lead to additional costs, which need to be considered when comparing both alternatives, especially when the cost difference between both models is not significant. 
If we consider the rigid case first, the total costs of problem one may be reduced by increasing the production rate of the second stage and by reducing the production rate of stages 1 and 3 for both the cases of equal- and unequal-sized batch shipments. A comparison with the results shown in table 2 indicates that this strategy shifts inventory from the first two buffer stocks to the last one, which is subject to lower inventory carrying charges in the present case. Further, it can be seen that the overall inventory in the system is reduced by moving the production rates closer to each other. Tables 5 and 6 show that varying the production rates with every shipment leads to a further reduction in total costs, although inventory is not necessarily reduced. This is due to the fact that it is beneficial to increase the lot size, which may lead to higher inventory carrying costs, but which reduces the number of lots in the planning period and consequently transportation and setup costs.

Problem two illustrates the effect of a variation of the inventory carrying charges on the selection of the production rate. An increase in $h_{s}$ leads to higher inventory carrying costs, which enhances the advantage that results from varying the production rate. Further, as keeping inventory in the third buffer stock is now more expensive than keeping inventory in the first two buffers, it is beneficial to vary the production rates to such an extent that inventory is reduced in the last buffer stock. As can be seen in tables 3 to 6 , the production rate of stage 3 is reduced further, which prevents that inventory is transferred too fast from buffer 2 to buffer 3 . In addition, the system produces with a smaller lot size, which reduces overall inventory in the system.

If the transportation costs per shipment $T_{s}$ are increased, the number of shipments $m$ is reduced, which, ceteris paribus, leads to higher inventory in the system (cf. problem 3). To compensate this increase, the production rate of the first batches is increased, which reduces inventory. This reaction is obvious, since it has been shown that waiting time-related inventory is especially problematic in case $m$ is high, but that the weight of the production rate of a particular batch is reduced as $m$ decreases (cf. appendices 2 to 5). A similar effect results from varying the setup costs $A_{s}$, which also impacts the number of batch shipments in the planning period and the necessity of varying $p_{s}$ (cf. problem 4$)$.

\section{Table 3}

The impact that results from varying the shape of the unit production cost function is illustrated in problems five and six. If the function is compressed, increasing or decreasing the 


\section{Conclusion}

Table 5

Table 6

This paper extended the work of Glock (2010a) to consider a multi-stage production system. This system was too investigated for equal and unequal sized shipments between successive stages for production rates that have to be fixed prior to the start of a production run or that may be varied during the production process. The results of the paper showed that

- allowing production rates to be varied reduces inventory in the system and results in lower total costs. The case where production rates may be varied for each batch produced leads to lower total costs than the case where the production rates have to be fixed before the start of a production run. Further, unequal-sized batch shipments lead to lower total costs than equal-sized batch shipments.

- system inventory may be reduced by bringing the production rates of subsequent stages of a serial production system closer to each other. This enables the system to process products smoothly without having to build up large buffer stocks in front of or in the back-end of bottleneck stations.

- varying production rates helps to shift inventory between buffer stocks, which enables the production planner to exploit different inventory carrying charges in the system. 
- the question whether the production rate of a production facility should be increased or reduced depends on (1) the number of batch shipments, (2) the production rates of the preceding and subsequent stage and (3) the inventory carrying charges applicable to the buffer stocks located in front of and in the back-end of the respective production stage.

Our research is especially important for companies facing high in-process inventories or bottleneck-problems. As could be shown, varying the production rates in a serial production system may significantly influence waiting time-related inventory and may thus help to control queues in front of subsequent production stages. This is consistent with the OPTPhilosophy (see for example Goldratt 1988), which aims on maximizing the throughput of the production system as a whole, instead of the throughput of a single machine, and the JITphilosophy, which aims on reducing in-process inventories.

One important aspect that has not been addressed in this paper is that varying the production rate may impact the quality of the product under consideration. Thus, with an increasing or a decreasing production rate, the production process may produce a higher or a lower percentage of defective products that need to be reworked or scrapped. Although the production cost function formulated in (5) may reflect this relation in part, more complex interdependencies between the production rate of a production facility and the quality of the products produced on this facility may prevail in practice. Consequently, it might be necessary to consider a separate quality cost function to account for scrap, rework or shortages as a result of a variation in the production rates. One approach could be to use an exponentially distributed random variable, which describes the point in time at which a production process goes out of control, and to establish a connection between the production rate of the production facility and the mean of the variable (see for example Lee and Rosenblatt 1987; Zhu et al. 2007). Thus, with an increase or a decrease of the production rate, the mean of the random variable would be changed, resulting in a lower or higher probability of the production process going out of control.

In order to increase the scope of our analysis, the model presented in this article could further be extended to include costs of changing the production rate or a different unit production cost function (see for example Khouja 1995; Moon and Christy 1998). Further, it would be interesting to permit a different number of batch shipments at each production stage, which has been shown to reduce total costs (Bogaschewsky et al. 2001).

\section{Appendices}




\section{Appendix 1}

If we consider three subsequent stages, we may differentiate between the four cases illustrated in table 7.

\section{Table 7}

While component II is always positive for cases 1 to 4 , component I adopts a positive value for $m=1$ and a negative value for $m>2$. It is obvious that in case of $m=1$, all four cost functions may be reduced by increasing $p_{s}$, while a reduction in $p_{s}$ leads to an increase in inventory carrying costs. Due to the fact that a variation in $p_{s}$ always leads to an increase in production costs, it cannot be optimal to produce with a production rate lower than $p_{s, 0}$ in case $m=1$.

In contrast, if $m=2$, component I reduces to zero in all four cost functions (cf. table 7). Thus, inventory carrying costs may be reduced by reducing component II. As can be seen, component II is independent of $p_{s}$ in case 2 , wherefore we can conclude that if $p_{s-1}<p_{s}>p_{s+1}$, it is not beneficial to produce with another production rate than $p_{s, 0}$, which would result in increased production costs, but not in lower inventory carrying costs. This effect is due to the fact that in case $p_{s-1}<p_{s}>p_{s+1}$ and $m=2$, a variation in $p_{s}$ leads to a decrease (increase) in $I^{\text {reg }}$ that is exactly compensated by an increase (decrease) in $I^{\text {wait }}$ (it can be seen in figure 1 by comparing the bold and dashed lines that a decrease in $p_{s}$ leads to a higher regular inventory and a lower inventory due to waiting times). This has been shown by Glock (2010a) in the context of a two-stage production system.

For the other three cases, i.e. $p_{s-1}>p_{s}>p_{s+1}, p_{s-1}>p_{s}<p_{s+1}$, and $p_{s-1}<p_{s}<p_{s+1}$, inventory carrying costs may be reduced by increasing $p_{s}$. This effect can be explained by analysing the variation of $I^{\text {reg }}$ and $I^{\text {wait }}$ for the case that $p_{s}$ is varied to $p_{s}$ '. It can be seen that (A-3) is positive for $m=2$ and $p_{s}{ }^{\prime}>p_{s}$, wherefore we may conclude that an increase in $p_{s}$ leads to a reduction in the regular inventory that compensates the increase in waiting time-related inventory. Thus, since $c_{p, s}\left(p_{s}\right)$ increases with an increasing or a decreasing production rate, it is optimal to produce with $p_{s}>p_{s, 0}$. 


$$
\Delta I^{\text {reg }}+\Delta I^{\text {wait }}= \begin{cases}\frac{D Q}{2 m}\left(\frac{1}{p_{s}}-\frac{1}{p_{s^{\prime}}}\right)\left(h_{s}(2-m)+m h_{s-1}\right) & p_{s-1}>p_{s}>p_{s+1} \\ \frac{D Q}{2 m}\left(\frac{1}{p_{s}}-\frac{1}{p_{s^{\prime}}}\right)\left(h_{s-1}+h_{s}\right)(2-m) & p_{s-1}<p_{s}>p_{s+1} \\ \frac{D Q}{2}\left(\frac{1}{p_{s}}-\frac{1}{p_{s^{\prime}}}\right)\left(h_{s-1}+h_{s}\right) & p_{s-1}>p_{s}<p_{s+1} \\ \frac{D Q}{2 m}\left(\frac{1}{p_{s}}-\frac{1}{p_{s^{\prime}}}\right)\left(h_{s-1}(2-m)+m h_{s}\right) & p_{s-1}<p_{s}<p_{s+1}\end{cases}
$$

Finally, for $m>2$, it can be seen that in case $p_{s-1}>p_{s}>p_{s+1}$, the second bracket term in (A-3) is positive for $m /(m-2)>h_{s} / h_{s-1}$. In this case, it is beneficial to increase $p_{s}$, which would lead to a positive value for the first bracket term and consequently lower inventory carrying costs. In contrast, if $m /(m-2)<h_{s} / h_{s-1}$, it is beneficial to reduce $p_{s}$.

For the case $p_{s-1}<p_{s}>p_{s+1}$, it can be seen that the third bracket term in (A-3) is always negative for $m>2$. Thus, inventory carrying costs can be reduced by reducing $p_{s}$, which leads to a positive value for $\Delta I^{\text {reg }}+\Delta I^{\text {wait }}$ in total. If $p_{s-1}>p_{s}<p_{s+1}$ holds, (A-3) illustrates that the sum of $\Delta I^{r e g}+\Delta I^{\text {wait }}$ is independent of $m$, and that the change in inventory which results from varying $p_{s}$ to $p_{s}$ ' may be increased by enhancing the production rate at stage $s$.

Finally, in case $p_{s-1}<p_{s}<p_{s+1}$, it can be seen that the second bracket term in (A-3) is positive for $m /(m-2)>h_{s-1} / h_{s}$. In this case, it is beneficial to increase $p_{s}$, which would lead to a positive value for the first bracket term and consequently lower inventory carrying costs. To the contrary, if $m /(m-2)<h_{s} / h_{s-1}$, it is beneficial to reduce $p_{s}$.

\section{Appendix 2}

In case $p_{s-1}>p_{s}>p_{s+1}$, inventory costs for three subsequent stages as given in (19) reduce to:

$$
\begin{aligned}
& \frac{D Q}{m^{2}}\left(\sum_{i=2}^{m-1}(\frac{3 h_{s}}{2 p_{i, s+1}}-\frac{h_{s-1}}{2 p_{i, s-1}}-\sum_{j=2}^{i} \frac{h_{s-1}}{p_{j, s-1}}+\overbrace{\sum_{j=2}^{i-1} \frac{h_{s-1}-h_{s}}{p_{j, s}}+\frac{3\left(h_{s-1}-h_{s}\right)}{2 p_{i, s}}}^{\mathrm{I}}+\sum_{j=2}^{i-1} \frac{h_{s}}{p_{j, s+1}})+\right. \\
& +\frac{1}{2}(\frac{h_{s-1}}{p_{1, s-1}}+\underbrace{\frac{(2 m-1) h_{s-1}+h_{s}}{p_{1, s}}}_{\text {II }}+\frac{h_{s}(2 m-1)}{p_{1, s+1}}-\frac{h_{s-1}}{p_{m, s-1}}+\underbrace{\frac{h_{s-1}-h_{s}}{p_{m, s}}}_{\text {III }}+\frac{h_{s}}{p_{m, s+1}})
\end{aligned}
$$

As can be seen, only component II depends on $p_{1, s}$, i.e. the production rate of the first batch produced on stage $s$. Since component II is always positive, inventory carrying costs may be reduced by increasing $p_{1, s}$. This result is obvious, as an increase in the production rate of the first batch leads to a faster inventory build-up and enables stage $s+1$ to initiate consumption earlier, which reduces inventory in the system. 
Looking at the production rate of the last batch, it can be seen in (A-4) that only component III depends on $p_{m, s}$. Due to the fact that III is positive for $h_{s-1}>h_{s}$ and negative for the opposite case, inventory carrying costs may be reduced by increasing $p_{m, s}$ if $h_{s-1}>h_{s}$ and vice versa. This result may be explained by the fact that a variation in $p_{m, s}$ leads to a shift of inventory between the buffer stocks in front of stages $s$ and $s+1$, which enables the system to take advantage of different inventory carrying charges along the supply chain.

Finally, considering component $\mathrm{I}$, it can be seen that an increase in $p_{j, s}$ for $j=2, \ldots, m-1$ leads to a reduction in inventory if $h_{s-1}>h_{s}$ and vice versa for the same reasons as explained above. Further, it becomes obvious that the sum function in the component leads to a higher weight for the production rate of earlier batches, which is due to the fact that the production rate of batch $j$ with $j>1$ influences the waiting times of all subsequent batch shipments. Thus, if $p_{j, s}$ is reduced, the reduction for $p_{j, s}$ is larger than for $p_{k, s}$ with $j<k$, and vice versa.

\section{Appendix 3}

In case $p_{s-1}>p_{s}<p_{s+1}$, inventory costs for three subsequent stages as given in (19) reduce to:

$$
\begin{aligned}
& \frac{D Q}{m^{2}}\left(\sum_{i=2}^{m-1}\left(-\sum_{j=2}^{i} \frac{h_{s-1}}{p_{j, s-1}}-\frac{h_{s-1}}{2 p_{i, s-1}}+\frac{\overbrace{h_{s-1}+h_{s}}^{2 p_{i, s}}+\sum_{j=2}^{i} \frac{h_{s-1}}{p_{j, s}}+\sum_{j=i}^{m-1} \frac{h_{s}}{p_{j, s}}}{2}-\frac{h_{s}}{2 p_{i, s+1}}-\sum_{j=i}^{m-1} \frac{h_{s}}{p_{j, s+1}}\right)+\right. \\
& +\frac{1}{2}(\frac{h_{s-1}}{p_{1, s-1}}+\underbrace{\frac{(2 m-1) h_{s-1}+h_{s}}{p_{1, s}}}_{\mathrm{II}}-\frac{h_{s}}{p_{1, s+1}}-\frac{h_{s-1}}{p_{m, s-1}}+\underbrace{\frac{h_{s-1}+(2 m-1) h_{s}}{p_{m, s}}}_{\mathrm{III}}+\frac{h_{s}}{p_{m, s+1}})
\end{aligned}
$$

In this case, component II and III, which are always positive, depend on $p_{1, s}$ and $p_{m, s}$, respectively. Thus, inventory carrying costs may be reduced by increasing $p_{1, s}$ and $p_{m, s}$, which leads to a lower value for components II and III. Further, it can be seen that component I is strictly positive and that it may be reduced by increasing $p_{j, s}$ for $j=2, \ldots, m-1$. Thereby, the sum function in component I leads to a higher weight for the production rate of earlier batches if $h_{s-1}>h_{s}$ and to a higher weight for the production rate of later batches if $h_{s-1}<h_{s}$. This may be explained by the fact that a faster production rate for later batches enables the system to initiate consumption earlier, which reduces inventory in front of stage $s+1$. In contrast, if earlier batches are produced with a higher production rate, waiting times are reduced for batches stored in the buffer stock in front of stage $s$. Thus, by varying the production rates of subsequent batches differently, the system may take advantage of different inventory carrying costs in the system. As a consequence, we may conclude that it is optimal to increase $p_{j, s}$ stronger than $p_{k, s}$ for $j<k$ if $h_{s-1}>h_{s}$ and vice versa. 


\section{Appendix 4}

In case $p_{s-1}<p_{s}>p_{s+1}$, inventory costs for three subsequent stages as given in (19) reduce to:

$$
\begin{aligned}
& \frac{D Q}{m^{2}}\left(\sum_{i=2}^{m-1}(\sum_{j=i}^{m-1} \frac{h_{s-1}}{p_{j, s-1}}+\frac{h_{s-1}}{2 p_{i, s-1}}+\frac{3 h_{s}}{2 p_{i, s+1}}-\overbrace{\frac{h_{s}+h_{s-1}}{2 p_{i, s}}-\sum_{j=i}^{m-1} \frac{h_{s-1}}{p_{j, s}}+\sum_{j=2}^{i} \frac{h_{s}}{p_{j, s}}}^{\mathrm{I}}+\sum_{j=2}^{i-1} \frac{h_{s}}{p_{j, s+1}})+\right. \\
& +\frac{1}{2}(\frac{h_{s-1}}{p_{1, s-1}}+\underbrace{\frac{h_{s}-h_{s-1}}{p_{1, s}}}_{\mathrm{II}}+\frac{h_{s}(2 m-1)}{p_{1, s+1}}+\frac{(2 m-1) h_{s-1}}{p_{m, s-1}}+\underbrace{\frac{h_{s-1}-h_{s}}{p_{m, s}}}_{\mathrm{III}}+\frac{h_{s}}{p_{m, s+1}})
\end{aligned}
$$

As can be seen in (A-6), component II is positive in case $h_{s}>h_{s-1}$ and negative for the opposite case. Thus, if $h_{s}>h_{s-1}$, it is optimal to increase $p_{1, s}$, while it is optimal to reduce $p_{1, s}$ if $h_{s}<h_{s-1}$. The opposite pertains for component III, which is positive for $h_{s-1}>h_{s}$ and negative for $h_{s-1}<h_{s}$. Thus, we conclude that it is optimal to increase $p_{\mathrm{m}, s}$ if $h_{s-1}>h_{s}$ and to reduce $p_{\mathrm{m}, s}$ if $h_{s-1}<h_{s}$.

As to the production rate for batches 2 to $m-1$, it can be seen that component $\mathrm{I}$ is strictly negative, wherefore inventory carrying costs may be reduced by reducing $p_{j, s}$ for $j=2, \ldots, m-1$. As explained above, the sum function in component I leads to a higher weight for the production rate of earlier batches if $h_{s-1}>h_{s}$ and to a higher weight for the production rate of later batches if $h_{s-1}<h_{s}$. As a consequence, we may conclude that it is optimal to reduce $p_{j, s}$ stronger than $p_{k, s}$ for $j<k$ if $h_{s-1}>h_{s}$ and vice versa.

\section{Appendix 5}

In case $p_{s-1}<p_{s}<p_{s+1}$, inventory costs for three subsequent stages as given in (19) reduce to:

$$
\begin{aligned}
& \frac{D Q}{m^{2}}\left(\sum_{i=2}^{m-1}(\sum_{j=i}^{m-1} \frac{h_{s-1}}{p_{j, s-1}}+\frac{h_{s-1}}{2 p_{i, s-1}}+\overbrace{\frac{h_{s}-h_{s-1}}{2 p_{i, s}}+\sum_{j=i}^{m-1} \frac{h_{s}-h_{s-1}}{p_{j, s}}}^{\mathrm{I}}-\frac{h_{s}}{2 p_{i, s+1}}-\sum_{j=i}^{m-1} \frac{h_{s}}{p_{j, s+1}})+\right. \\
& +\frac{1}{2}(\frac{h_{s-1}}{p_{1, s-1}}+\underbrace{\frac{h_{s}-h_{s-1}}{p_{1, s}}}_{\mathrm{II}}-\frac{h_{s}}{p_{1, s+1}}+\frac{(2 m-1) h_{s-1}}{p_{m, s-1}}+\underbrace{\frac{h_{s-1}+(2 m-1) h_{s}}{p_{m, s}}}_{\mathrm{III}}+\frac{h_{s}}{p_{m, s+1}})
\end{aligned}
$$

Component II of (A-7) is positive in case $h_{s}>h_{s-1}$ and negative for the opposite case. Thus, if $h_{s}>h_{s-1}$, it is optimal to increase $p_{1, s}$, while it is optimal to reduce $p_{1, s}$ if $h_{s}<h_{s-1}$. In contrast, component III is always positive, wherefore it is inventory carrying costs may be reduced by increasing $p_{m, s}$.

Finally, component I is positive for $h_{s}>h_{s-1}$ and negative for the opposite case. Thus, in case $h_{s}>h_{s-1}$, it is optimal to increase $p_{j, s}$ and to reduce $p_{j, s}$ if $h_{s}<h_{s-1}$. Again, the sum function in component I leads to a higher weight for the production rate of later batches, wherefore it is beneficial to vary $p_{j, s}$ stronger than $p_{k, s}$ for $j>k$. 


\section{Acknowledgement}

The author is grateful to the anonymous referees for their valuable comments and suggestions on an earlier version of this paper. The author further wishes to thank the editorial team and the reviewers for the fast reviewing process.

\section{Literature}

Bogaschewsky R W, Buscher U D, and Lindner G (2001). Optimizing multi-stage production with constant lot size and varying number of unequal sized batches. Omega 29: 183-191.

Buzacott J A and Ozkarahan I A (1983). One- and Two-Stage Scheduling of Two Products with Distributed Inserted Idle Time: The Benefits of a Controllable Production Rate. Naval Research Logistics Quarterly 30: 675-696.

Chang J H, and Chiu H N (2005). A comprehensive review of lot streaming. International Journal of Production Research 43: 1515-1536.

Chiang S Y, Kuo C T and Meerkov S M (2000). DT-Bottlenecks in Serial Production Lines: Theory and Application. IEEE Transactions on Robotics and Automation 16: 567-580.

Cochran J K and Kim S S (1998). Optimum junction point location and inventory levels in serial hybrid push/pull production systems. International Journal of Production Research 36: 1141-1155.

Eiamkanchanalai S and Banerjee A (1999). Production lot sizing with variable production rate and explicit idle capacity cost. International Journal of Production Economics 59: 251-259.

Gill P E, Murray W and Wright M H (1986). Practical Optimization. Amsterdam: Elsevier.

Glock C H (2010a). Batch sizing with controllable production rates. International Journal of Production research 48: 5925-5942..

Glock C H (2010b). Supply chain coordination via integrated inventory models: a review, working paper, University of Wuerzburg.

Goldratt E M (1988). Computerized shop floor scheduling. International Journal of Production Research 26: 443-455.

Goyal S K (1976). Note on manufacturing cycle time determination for a multi-stage economic production quantity model. Management Science 23: 332-333.

Goyal S K (1977a). Economic batch quantity in a multi-stage production system. International Journal of Production Research 16: 267-273.

Goyal S K (1977b). Determination of Optimum Production Quantity for a Two-Stage Production System. Operations Research Quarterly 28: 865-870. 
Higgins P, Le Roy P, and Tierney L (1996). Manufacturing Planning and Control. London: Chapman \& Hall.

Hsiao Y-C, Lin Y, and Huang Y-K (2010). Optimal multi-stage logistic and inventory policies with production bottleneck in a serial supply chain. International Journal of Production Economics 124: 408-413.

Khouja M (1995). The economic production lot size model under volume flexibility. Computers and Operations Research 22: 513-523.

Kuo C T, Lim J T, and Meerkov S M (1996). Bottlenecks in serial production lines: A system-theoretic approach. Mathematical Problems in Engineering 2: 233-276.

Lee H L, Padmanabhan V, and Whang S (1997). The Bullwhip Effect in Supply Chains. Sloan Management Review 38: 93-102.

Lee H L, and Rosenblatt M J (1987). Simultaneous determination of production cycle and inspection schedules in a production system. Management Science 33: 1125-1136.

Moon D H and Christy D P (1998). Determination of optimal production rates on a single facility with dependent mold lifespan. International Journal of Production Economics 54: 2940.

Schweitzer P J and Seidmann A (1991). Optimizing processing rates for flexible manufacturing systems. Management Science 37: 454-466.

Szendrovits A Z (1975). Manufacturing cycle time determination for a multi-stage economic production quantity model. Management Science 22: 298-308.

Szendrovits A Z, and Drezner Z (1980). Optimizing Multi-Stage Production with Constant Lot Size and Varying Numbers of Batches. Omega 8: 623-629.

Zhu K, Zhang R Q, and Tsung F (2007). Pushing Quality Improvement Along the Supply Chain. Management Science 53: 421-436. 


\section{Table 1}

\begin{tabular}{|c|c|c|c|c|c|c|}
\hline$\#$ & $S$ & $\boldsymbol{h}_{\mathrm{s}}$ & $A_{s}$ & $T_{s}$ & $\left\{a_{0, s}, a_{1, s}, a_{2, s}\right\}$ & $\left\{p_{s, \min }, p_{s, \max }\right\}$ \\
\hline \multirow{3}{*}{1} & 1 & 3 & 250 & 20 & $\{1 / 6000,1 / 12,11\}$ & $\{230,300\}$ \\
\hline & 2 & 3 & 275 & 30 & $\{1 / 7000,2 / 35,6.5\}$ & $\{170,250\}$ \\
\hline & 3 & 2 & 200 & 25 & $\{1 / 9000,1 / 15,10.5\}$ & $\{270,320\}$ \\
\hline \multirow{3}{*}{2} & 1 & 3 & 250 & 20 & $\{1 / 6000,1 / 12,11\}$ & $\{230,300\}$ \\
\hline & 2 & 5 & 275 & 30 & $\{1 / 7000,2 / 35,6.5\}$ & $\{170,250\}$ \\
\hline & 3 & 10 & 200 & 25 & $\{1 / 9000,1 / 15,10.5\}$ & $\{270,320\}$ \\
\hline \multirow{3}{*}{3} & 1 & 3 & 250 & 40 & $\{1 / 6000,1 / 12,11\}$ & $\{230,300\}$ \\
\hline & 2 & 3 & 275 & 60 & $\{1 / 7000,2 / 35,6.5\}$ & $\{170,250\}$ \\
\hline & 3 & 2 & 200 & 50 & $\{1 / 9000,1 / 15,10.5\}$ & $\{270,320\}$ \\
\hline \multirow{3}{*}{4} & 1 & 3 & 125 & 20 & $\{1 / 6000,1 / 12,11\}$ & $\{230,300\}$ \\
\hline & 2 & 3 & 137.5 & 30 & $\{1 / 7000,2 / 35,6.5\}$ & $\{170,250\}$ \\
\hline & 3 & 2 & 100 & 25 & $\{1 / 9000,1 / 15,10.5\}$ & $\{270,320\}$ \\
\hline \multirow{3}{*}{5} & 1 & 3 & 250 & 20 & $\{1 / 600,10 / 12,105.17\}$ & $\{230,300\}$ \\
\hline & 2 & 3 & 275 & 30 & $\{1 / 700,20 / 35,58.06\}$ & $\{170,250\}$ \\
\hline & 3 & 2 & 200 & 25 & $\{1 / 900,10 / 15,100.54\}$ & $\{270,320\}$ \\
\hline \multirow{3}{*}{6} & 1 & 3 & 250 & 20 & $\{1 / 60,100 / 12,1042.67\}$ & $\{230,300\}$ \\
\hline & 2 & 3 & 275 & 30 & $\{1 / 70,200 / 35,572.34\}$ & $\{170,250\}$ \\
\hline & 3 & 2 & 200 & 25 & $\{1 / 90,100 / 15,1000.54\}$ & $\{270,320\}$ \\
\hline \multirow{3}{*}{7} & 1 & 3 & 250 & 20 & $\{1 / 6000,1 / 12,11\}$ & $\{200,320\}$ \\
\hline & 2 & 3 & 275 & 30 & $\{1 / 7000,2 / 35,6.5\}$ & $\{150,270\}$ \\
\hline & 3 & 2 & 200 & 25 & $\{1 / 9000,1 / 15,10.5\}$ & $\{250,350\}$ \\
\hline
\end{tabular}


Table 2

\begin{tabular}{|c|c|c|c|c|c|c|c|c|c|c|c|c|c|c|}
\hline \# & \multicolumn{7}{|c|}{ equal-sized batch shipments } & \multicolumn{7}{|c|}{ unequal-sized batch shipments } \\
\hline 2 & 4 & 137.14 & $\{250,200,300\}$ & 205.71 & 228.57 & 571.42 & 16817.3 & 4 & 152.69 & $\{250,200,300\}$ & 182.27 & 189.88 & 521.68 & 15295.2 \\
\hline 4 & 4 & 192.74 & $\{250,200,300\}$ & 289.11 & 321.24 & 803.09 & 8743.52 & 4 & 216.41 & $\{250,200,300\}$ & 258.35 & 269.13 & 739.41 & 7991.57 \\
\hline 5 & 5 & 258.99 & $\{250,200,300\}$ & 336.68 & 388.48 & 1035.94 & 10955.2 & 5 & 291.54 & $\{250,200,300\}$ & 287.86 & 316.64 & 979.82 & 10006.7 \\
\hline 6 & 5 & 258.99 & $\{250,200,300\}$ & 336.68 & 388.48 & 1035.94 & 10949.5 & 5 & 291.54 & $\{250,200,300\}$ & 287.86 & 316.64 & 979.82 & 10001.0 \\
\hline
\end{tabular}


Table 3

\begin{tabular}{|c|l|l|l|c|c|c|c|}
\hline$\#$ & $m^{*}$ & $Q^{*}$ & $\multicolumn{1}{c|}{p_{1}, \ldots, p_{S}}^{*}$ & $I_{1}$ & $I_{2}$ & \multicolumn{1}{c|}{$I_{3}$} & $T C^{*}$ \\
\hline 1 & 7 & 332.88 & $\{244.30,244.30,270\}$ & 194.66 & 240.98 & 1224.09 & 9764.98 \\
\hline 2 & 4 & 143.19 & $\{244.62,235.99,270\}$ & 157.03 & 170.79 & 583.35 & 16476.0 \\
\hline 3 & 5 & 337.01 & $\{245.84,248.84,270\}$ & 274.17 & 310.98 & 1310.61 & 11025.5 \\
\hline 4 & 4 & 209.50 & $\{241.95,238.63,285.58\}$ & 222.49 & 255.55 & 864.08 & 8440.81 \\
\hline 5 & 5 & 263.2 & $\{248.85,206.48,298\}$ & 320.05 & 372.38 & 1051.03 & 10885.8 \\
\hline 6 & 5 & 258.42 & $\{249.88,200.68,299.81\}$ & 334.93 & 386.78 & 1037.53 & 10942.3 \\
\hline 7 & 7 & 336.81 & $\{244.48,244.48,259.96\}$ & 196.81 & 226.10 & 1221.3 & 9757.59 \\
\hline
\end{tabular}


Table 4

\begin{tabular}{|c|l|l|c|c|c|c|c|}
\hline$\#$ & $m^{*}$ & \multicolumn{1}{|c|}{$Q^{*}$} & $\left\{p_{1}, \ldots, p_{S}\right\}^{*}$ & $I_{1}$ & $I_{2}$ & \multicolumn{1}{|c|}{$I_{3}$} & \multicolumn{1}{|c|}{$T C^{*}$} \\
\hline 1 & 6 & 318.542 & $\{249.71,228.04,275.30\}$ & 228.04 & 234.32 & 1018.85 & 9157.69 \\
\hline 2 & 4 & 157.43 & $\{250.96,220.61,270\}$ & 171.08 & 170.24 & 514.63 & 15051.3 \\
\hline 3 & 4 & 319.32 & $\{252.71,228.28,284.91\}$ & 337.09 & 333.94 & 1068.16 & 10308.6 \\
\hline 4 & 4 & 224.06 & $\{251.48,222.43,288.70\}$ & 241.74 & 241.43 & 753.64 & 7869.0 \\
\hline 5 & 5 & 294.24 & $\{249.73,204.40,297.75\}$ & 282.35 & 306.84 & 985.46 & 9970.88 \\
\hline 6 & 5 & 291.83 & $\{249.97,200.47,299.77\}$ & 287.24 & 315.56 & 980.46 & 9997.18 \\
\hline 7 & 6 & 318.52 & $\{249.71,228.04,275.30\}$ & 228.04 & 234.32 & 1018.85 & 9157.69 \\
\hline
\end{tabular}


Table 5

\begin{tabular}{|c|c|c|c|c|c|c|}
\hline$\#$ & \begin{tabular}{|l|l}
$s$ & $r$
\end{tabular} & $m^{*}$ & $Q^{*}$ & $\left\{p_{1, \mathrm{~s}}, \ldots, p_{m, s}\right\}^{*}$ & $I_{\mathrm{S}}$ & $T C^{*}$ \\
\hline 1 & \begin{tabular}{|l|}
1 \\
2 \\
3
\end{tabular} & 7 & 333.67 & $\begin{array}{l}\{253.34,233.36,237.22,240.84,244.22,247.42,250.47\} \\
\{250,247.82,244.43,240.89,237.16,233.24,250\} \\
\{298.80,270,270,270,270,270,301.19\}\end{array}$ & \begin{tabular}{l|}
181.91 \\
248.81 \\
1227.06
\end{tabular} & 9720.58 \\
\hline 2 & \begin{tabular}{|l|}
1 \\
2 \\
3
\end{tabular} & 4 & 143.39 & $\begin{array}{l}\{252.53,235.45,241.71,247.36\} \\
\{230.66,234.29,237.74,241.03\} \\
\{304.35,270,270,295.38\}\end{array}$ & $\begin{array}{l}155.61 \\
168.86 \\
583.73\end{array}$ & 16415.8 \\
\hline 3 & \begin{tabular}{l|}
1 \\
2 \\
3
\end{tabular} & 5 & 337.48 & $\begin{array}{l}\{254.63,235.95,241.65,246.27,250.56\} \\
\{250,245.92,241.36,236.44,250\} \\
\{298.15,270,270,270,301.72\}\end{array}$ & \begin{tabular}{l|}
264.02 \\
316.73 \\
1312.69
\end{tabular} & 10970.5 \\
\hline 4 & \begin{tabular}{l|} 
\\
2 \\
3
\end{tabular} & 4 & 211.43 & $\begin{array}{l}\{253.35,230.47,239.14,247.57\} \\
\{237.96,235.99,235.44,237.85\} \\
\{298.71,270.09,270.01,301.48\}\end{array}$ & $\begin{array}{l}216.48 \\
250.58 \\
861.63\end{array}$ & 8401.13 \\
\hline 5 & \begin{tabular}{l|}
1 \\
2 \\
3
\end{tabular} & 5 & 263.53 & $\begin{array}{l}\{250.38,247.29,248.07,248.85,249.62\} \\
\{206.49,206.49,206.49,206.49,206.49\} \\
\{299.87,296.91,296.63,296.35,300.13\}\end{array}$ & \begin{tabular}{|l|}
319.08 \\
371.51 \\
1051.19
\end{tabular} & 10880.8 \\
\hline 6 & \begin{tabular}{|l|}
1 \\
2 \\
3
\end{tabular} & 5 & 259.45 & $\begin{array}{l}\{250.04,249.74,249.81,249.89,249.96\} \\
\{200.68,200.68,200.68,200.68,200.68\} \\
\{299.99,299.70,299.68,299.65,300.01\}\end{array}$ & \begin{tabular}{|l|}
334.84 \\
386.70 \\
1037.54
\end{tabular} & 10941.8 \\
\hline 7 & \begin{tabular}{l|}
1 \\
2 \\
3
\end{tabular} & 7 & 342.59 & $\begin{array}{l}\{253.21,233.10,236.32,240.16,244.60,249.01,251.99\} \\
\{251.99,248.40,244.30,242.37,234.96,233.41,255.07\} \\
\{297.52,250,250,250,250,250,299.56\}\end{array}$ & $\begin{array}{l}184.34 \\
216.12 \\
1223.63\end{array}$ & 9668.39 \\
\hline
\end{tabular}


Table 6

\begin{tabular}{|c|c|c|c|c|c|c|}
\hline \# & $s$ & $m^{*}$ & $Q^{*}$ & $\left\{p_{1, \mathrm{~s}}, \ldots, p_{m, s}\right\}^{*}$ & $I_{\mathrm{S}}$ & $T C^{*}$ \\
\hline \multirow{3}{*}{1} & 1 & & & $\{253.05,250.52,249.31,249.12,249.91,251.84\}$ & 235.45 & \\
\hline & 2 & 6 & 331.13 & $\{212.22,221.82,228.77,232.45,233.27,231.97\}$ & 238.62 & 9109.74 \\
\hline & 3 & & & $\{320,270,270,270,270,287.42\}$ & 1063.12 & \\
\hline \multirow{3}{*}{2} & 1 & & & $\{252.30,250.80,250.53,251.45\}$ & 170.53 & \\
\hline & 2 & 4 & 157.68 & $\{212.41,219.83,224.13,223.98\}$ & 166.08 & 15019.4 \\
\hline & 3 & & & $\{320,270,270,271.14\}$ & 515.85 & \\
\hline \multirow{3}{*}{3} & 1 & & & $\{254.13,251.65,250.75,251.15,252.92\}$ & 302.33 & \\
\hline & 2 & 5 & 357.87 & $\{215.14,224.85,231.38,233.92,233.62\}$ & 299.62 & 10267 \\
\hline & 3 & & & $\{320,270,270,270,286.53\}$ & 1157.88 & \\
\hline \multirow{3}{*}{4} & 1 & & & $\{253.29,251.39,251.10,252.31\}$ & 241.49 & \\
\hline & 2 & 4 & 225.13 & $\{212.28,221.06,226.14,227.70\}$ & 237.5 & 7842.56 \\
\hline & 3 & & & $\{320,279.67,277.74,291.71\}$ & 752.92 & \\
\hline \multirow{3}{*}{5} & 1 & & & $\{250.22,249.59,249.26,249.21,249.43,249.91\}$ & 255.31 & \\
\hline & 2 & 6 & 316.46 & $\{201.43,203.39,205.29,207.11,208.78,209.80\}$ & 281.62 & 9950.47 \\
\hline & 3 & & & $\{320,273.12,282.57,293,296.68,298.83\}$ & 1051.1 & \\
\hline \multirow{3}{*}{6} & 1 & & & $\{250.03,249.96,249.93,249.94,249.99\}$ & 286.86 & \\
\hline & 2 & 5 & 292.12 & $\{200.16,200.42,200.66,200.89,201.09\}$ & 314.16 & 9993.35 \\
\hline & 3 & & & $\{303.72,298.19,299.19,299.65,299.88\}$ & 981.26 & \\
\hline \multirow{3}{*}{7} & 1 & & & $\{253,250.08,248.68,248.54,249.53,251.71\}$ & 238.70 & \\
\hline & 2 & 6 & 332.77 & $\{211.85,220.15,225.64,229.21,231.26,231\}$ & 235.21 & 9093.35 \\
\hline & 3 & & & $\{350,271.87,250,250,264.69,287.77\}$ & 1054.6 & \\
\hline
\end{tabular}


Table 7

\begin{tabular}{|c|c|c|}
\hline \# & relation & inventory costs \\
\hline 1 & $\mathrm{p}_{\mathrm{s}-1}>\mathrm{p}_{\mathrm{s}}>\mathrm{p}_{\mathrm{s}+1}$ & $\frac{D Q}{2 m}(\underbrace{\left(\frac{h_{s-1}}{p_{s-1}}+\frac{h_{s}}{p_{s}}\right)(2-m)}_{\mathrm{I}}+\underbrace{m\left(\frac{h_{s-1}}{p_{s}}+\frac{h_{s}}{p_{s+1}}\right)}_{\mathrm{II}})$ \\
\hline 2 & $\mathrm{p}_{\mathrm{s}-1}<\mathrm{p}_{\mathrm{s}}>\mathrm{p}_{\mathrm{s}+1}$ & $\frac{D Q}{2 m}(\underbrace{\frac{h_{s-1}+h_{s}}{p_{s}}(2-m)}_{\mathrm{I}}+m(\underbrace{\left.\left(\frac{h_{s-1}}{p_{s-1}}+\frac{h_{s}}{p_{s+1}}\right)\right)}_{\mathrm{II}}$ \\
\hline 3 & $\mathrm{p}_{\mathrm{s}-1}>\mathrm{p}_{\mathrm{s}}<\mathrm{p}_{\mathrm{s}+1}$ & 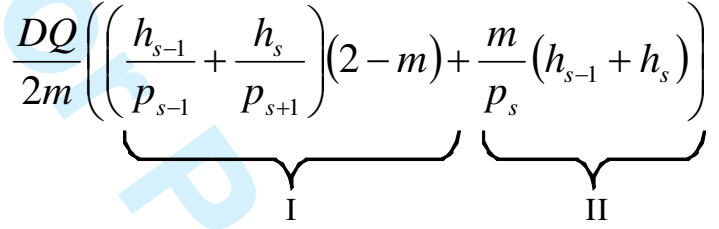 \\
\hline 4 & $\mathrm{p}_{\mathrm{s}-1}<\mathrm{p}_{\mathrm{s}}<\mathrm{p}_{\mathrm{s}+1}$ & 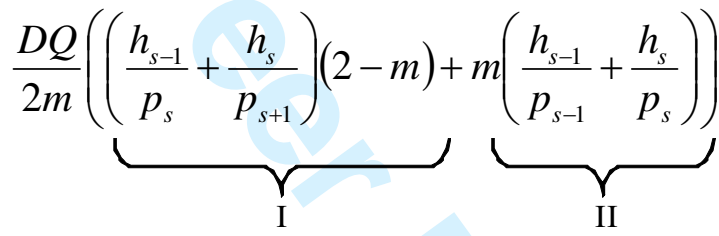 \\
\hline
\end{tabular}




\section{Captions for Figures and Tables}

Figure 1: Inventory-time plots for the case of equal-sized batch shipments

Figure 2: A three-stage production system with two buffer stocks

Figure 3: Inventory-time plots for the case of unequal-sized batch shipments

Table 1: Test problems used for computational experimentation

Table 2: Computational results for the case of fixed production rates

Table 3: Computational results for the rigid case with equal-sized batch shipments

Table 4: Computational results for the rigid case with unequal-sized batch shipments

Table 5: Computational results for the flexible case with equal-sized batch shipments

Table 6: Computational results for the flexible case with unequal-sized batch shipments

Table 7: Inventory carrying cost for alternative ratios of the production rates 


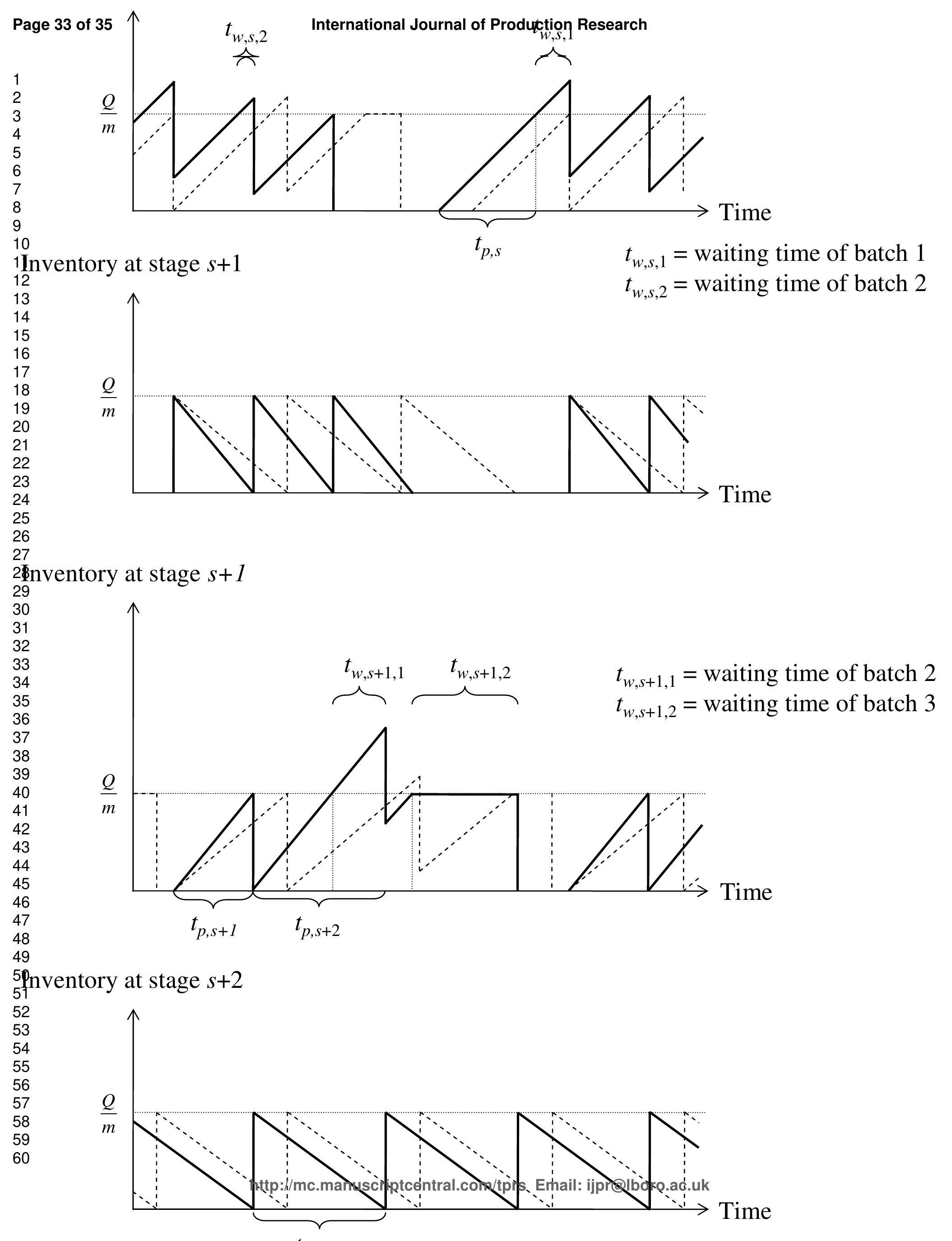




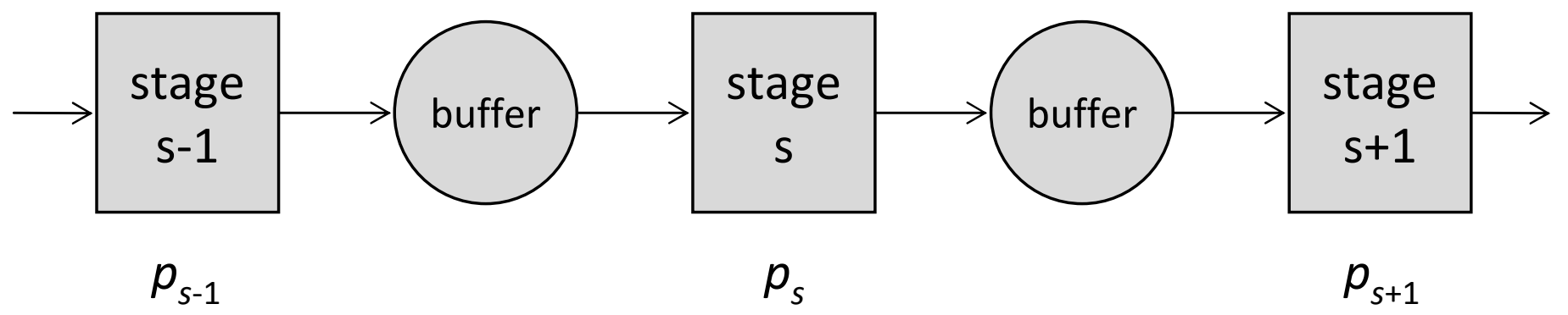




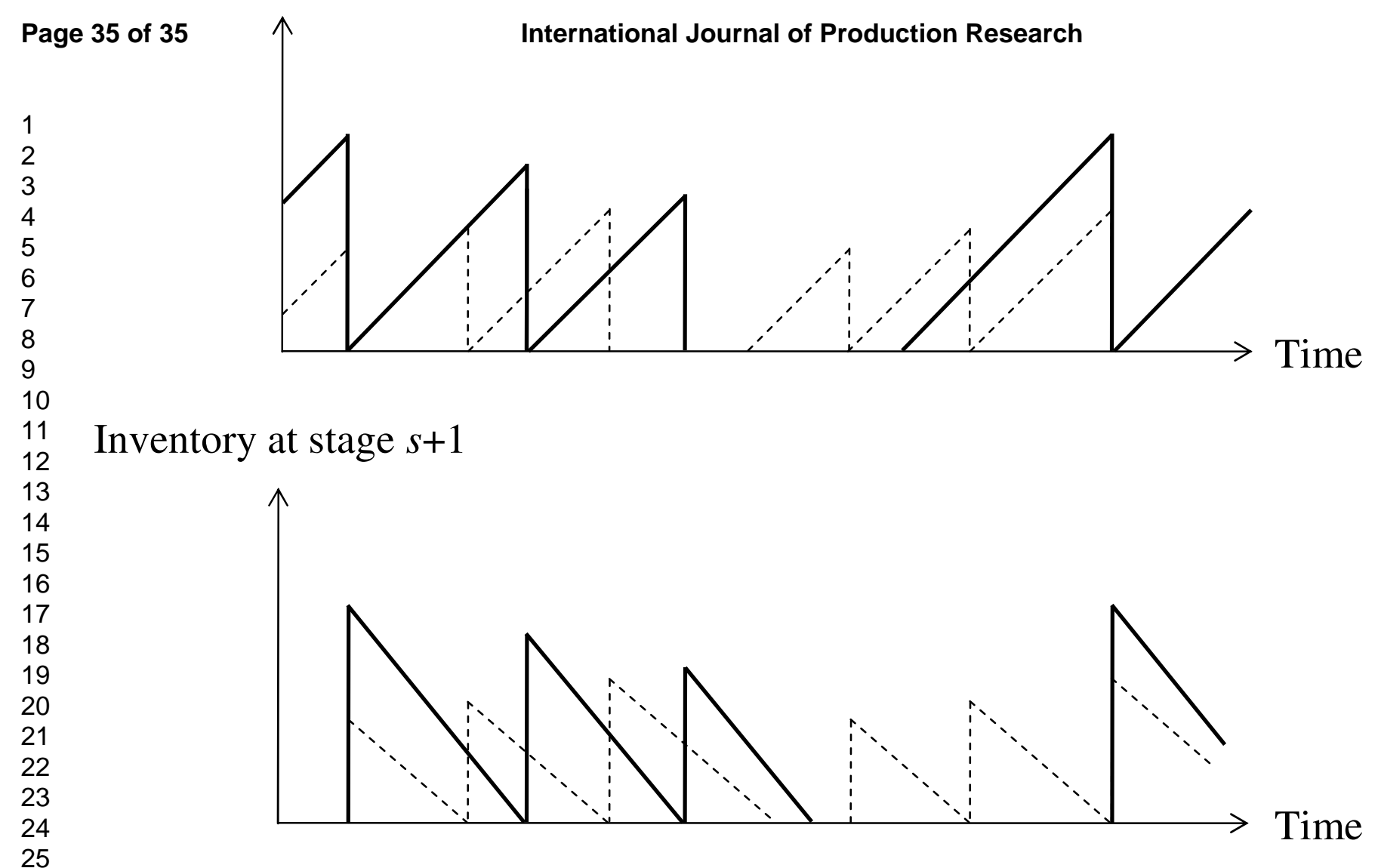

Inventory at stage $s+1$

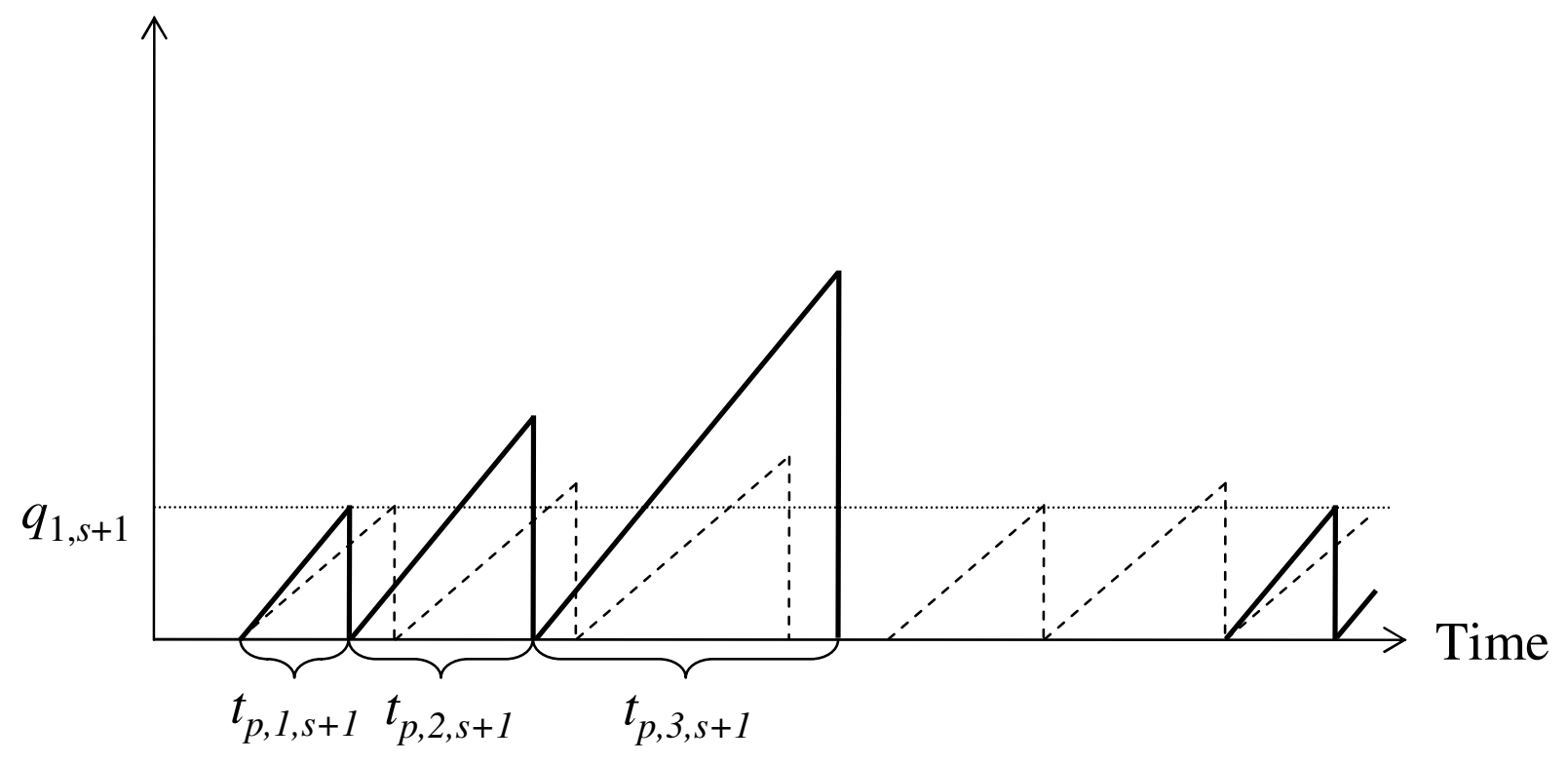

Inventory at stage $s+2$

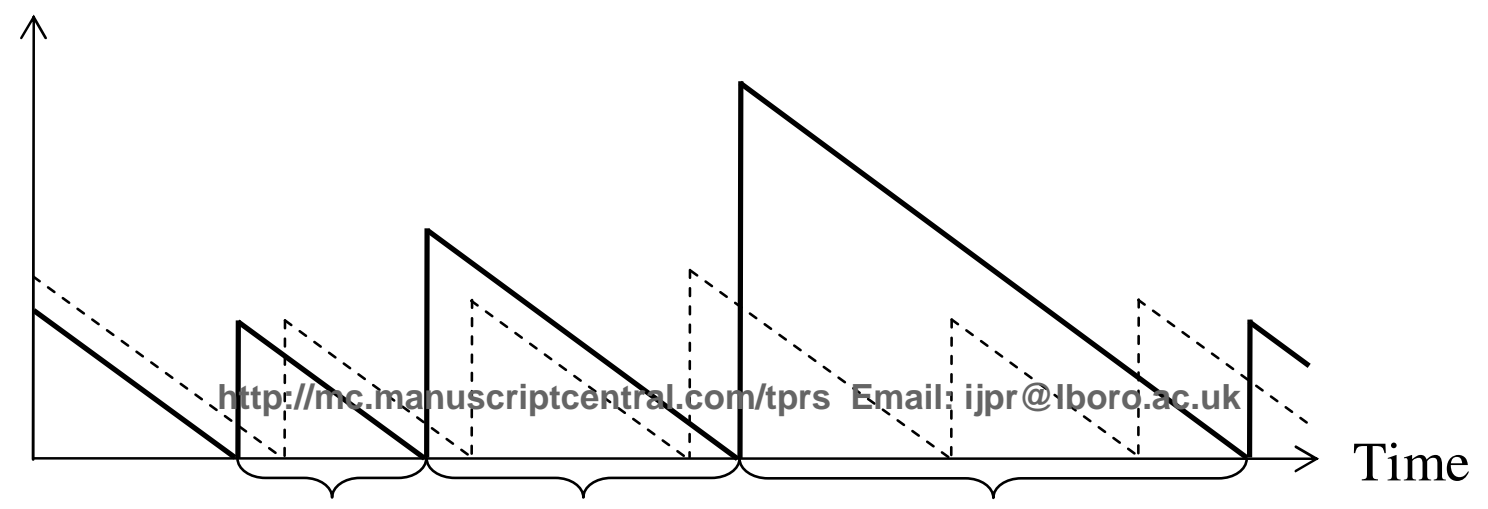

